\title{
Riesz completions, functional representations, and anti-lattices
}

\author{
Anke Kalauch, Bas Lemmens and Onno van Gaans
}

October 20, 2010

\begin{abstract}
We show that the Riesz completion of an Archimedean partially ordered vector space $X$ with unit can be represented as a norm dense Riesz subspace of the smallest functional representation of $X$. This yields a convenient way to compute the Riesz completion. To illustrate the method, the Riesz completions of Lorentz cones, cones of symmetric positive semidefinite matrices, and polyhedral cones are determined. We use the representation to investigate the existence of nontrivial disjoint elements and link the absence of such elements to the notion of anti-lattice. One of the results is a geometric condition on the dual cone of a finite dimensional partially ordered vector space $X$ that ensures that $X$ is an anti-lattice.
\end{abstract}

\section{Introduction}

A useful tool in the analysis of partially ordered vector spaces is the Riesz completion, as introduced by van Haandel [9]. The Riesz completion of a partially ordered vector space is the smallest Riesz space into which the partially ordered vector space can be bipositively embedded as an order-dense subspace. It provides a tool to analyze generalizations of a variety of concepts from Riesz space theory, such as disjointness, bands, and ideals, in the setting of partially ordered vector spaces $[6,7,8]$. The construction of the Riesz completion as given by van Haandel [9] relies on algebraic techniques and is rather elaborate. For many practical purposes one requires a simpler, more direct construction.

A very direct method to associate a Riesz space to a partially ordered vector space is based on the "functional representation method", which, roughly speaking, represents each Archimedean partially ordered vector space $X$ with order unit as a subspace of $C(\Xi)$, where $C(\Xi)$ is the space of continuous functions on a compact Hausdorff space $\Xi$. There are various natural ways to do this, see e.g. Krein [14] and Nachbin [15]. For our purpose we need to follow Kadison [11] and consider the smallest functional representation. In fact, we shall see that the Riesz completion of an Archimedean partially ordered vector space $X$ with order unit is a norm dense Riesz subspace of the smallest functional representation $C(\Xi)$ of $X$.

This result is implicit in the work of van Haandel [9, Theorems 5.8 and 5.10]. By making the connection with the functional representation we provide a more intuitive geometric proof of this result, see Theorem 10. We consider the smallest functional representation $C(\Omega)$ of the Riesz completion of $X$ and 
show that $\Xi$ and $\Omega$ are homeomorphic as main step in the proof, whereas van Haandel's proofs rely on a detailed analysis of Riesz* homomorphisms. Our approach clarifies the connections between the works of Kadison [11], Alfsen [2] and van Haandel [9].

To illustrate the power of the functional representation we compute the Riesz completion for a variety of interesting Archimedean partially ordered vector spaces including, the Lorentz cones, the cones of symmetric positive semidefinite matrices, and polyhedral cones. We also use the method to find sufficient conditions for an Archimedean partially ordered vector space to be an anti-lattice. Recall that a partially ordered vector space $X$ is an anti-lattice if $x$ and $y$ in $X$ are comparable, whenever $x$ and $y$ have a greatest lower bound. We show that $X$ is an anti-lattice if, and only if, there are no non-trivial disjoint elements in the cone, see Theorem 14. Furthermore an example of an anti-lattice with disjoint elements is given, which shows that there are anti-lattices that possess some lattice structure. In addition, we present for finite dimensional partially ordered vector spaces a geometric condition on the dual cone that ensures that there are no non-trivial disjoint elements, see Theorem 15. Furthermore, we prove that the space $\left(V, \mathrm{Pos}_{n}\right)$ consisting of symmetric matrices with partial ordering induced by the cone of positive semi-definite matrices, has no non-trivial disjoint elements. As a consequence $\left(V, \mathrm{Pos}_{n}\right)$ is an anti-lattice, which reproduces a well-known result by Kadison [12].

\section{Preliminaries}

Let $X$ be a real vector space containing a cone $K$; so $K$ is convex, $\lambda K \subseteq K$ for all $\lambda \geq 0$, and $K \cap(-K)=\{0\}$. The cone $K$ induces a partial ordering $\leq$ in $X$ by $x \leq y$ if $y-x \in K$. Recall that $K$ is Archimedean if for any $x, y \in X$ with $n x \leq y$ for all $n \in \mathbb{N}$ we have $x \leq 0$. In that case $(X, K)$ is called an Archimedean partially ordered vector space. Throughout the exposition we assume that $(X, K)$ has an order unit $u \in K$, i.e., for each $x \in X$ there exists $\alpha \geq 0$ such that $-\alpha u \leq x \leq \alpha u$.

As usual [17, I.9.1], an order unit $u \in K$ gives rise to a norm $\|\cdot\|_{u}$ on $X$ by

$$
\|x\|_{u}=\inf \{\alpha>0:-\alpha u \leq x \leq \alpha u\} .
$$

This norm is called an order unit norm. Note that $u$ is in the interior of $K$ and each linear functional $\varphi: X \rightarrow \mathbb{R}$ with $\varphi(K) \subseteq[0, \infty)$ is continuous, as $|\varphi(x)| \leq \varphi(u)$ for all $x \in X$ with $\|x\|_{u} \leq 1$. As $K$ has non-empty interior the set $K^{*}=\{\varphi: X \rightarrow \mathbb{R}: \varphi$ is a positive linear functional $\}$ is a cone in the dual space $X^{*}$ of $\left(X,\|\cdot\|_{u}\right)$.

Denote

$$
\Sigma=\left\{\varphi \in K^{*}: \varphi(u)=1\right\} .
$$

By the Banach-Alaoglu theorem the closed unit ball $B^{*}$ of $X^{*}$ is weak-* compact. As $\Sigma$ is a weak-* closed subset of $B^{*}$, is is weak-* compact. Let

$$
\Lambda=\{\varphi \in \Sigma: \varphi \text { is an extreme point of } \Sigma\} .
$$

Recall that $\varphi \in \Sigma$ is extreme if $\varphi$ is not in the (relative) interior of a linesegment in $\Sigma$. In general, $\Lambda$ need not be weak-* closed, not even if $X$ is finite 
dimensional. We let $\bar{\Lambda}$ denote the weak-* closure of $\Lambda$ in $\Sigma$, so $\bar{\Lambda}$ is a compact Hausdorff space. We write $\Phi: X \rightarrow C(\bar{\Lambda})$ to denote the linear map

$$
(\Phi(x))(\varphi)=\varphi(x) \quad \text { for } \varphi \in \bar{\Lambda} .
$$

In the sequel we will use $\Sigma, \Lambda$, and $\Phi$ as defined above for the Archimedean partially ordered vector space $(X, K)$ with an order unit $u$ under consideration.

Recall that a linear map $\Psi: X \rightarrow Y$, where $X$ and $Y$ are partially ordered vector spaces, is called bipositive if for all $x \in X, x \geq 0$ is equivalent to $\Psi(x) \geq 0$.

The following theorem is due to Kadison [11, Theorem 2.1]. For completeness we provide the proof here.

Theorem 1 (Kadison). If $(X, K)$ is an Archimedean partially ordered vector space with an order unit $u$, then $\Phi: X \rightarrow C(\bar{\Lambda})$ is a bipositive linear map, which maps $u$ to the constant 1 function in $C(\bar{\Lambda})$.

Proof. It is clear that $\Phi$ is a positive linear map from $X$ into $C(\bar{\Lambda})$ which maps $u$ to the constant 1 function. To prove bipositivity, we first show that $K$ is $\|\cdot\|_{u}$-closed. If $\left(x_{n}\right)_{n}$ in $K$ and $x \in X$ are such that $\left\|x_{n}-x\right\|_{u} \rightarrow 0$, then $\left\|x_{n_{k}}-x\right\|_{u}<1 / k$ for a suitable subsequence, so $x \geq x_{n_{k}}-(1 / k) u \geq-(1 / k) u$ for all $k$. Hence $x \in K$, as $X$ is Archimedean. Consequently, if $x \in X \backslash K$, then there exists a continuous linear functional $\varphi: X \rightarrow \mathbb{R}$ such that $\varphi \in K^{*}$ and $\varphi(x)<0$. Thus, $x \in K$ is equivalent to $\varphi(x) \geq 0$ for all $\varphi \in K^{*}$.

By the Krein-Milman theorem, $\Sigma$ equals the weak-* closed convex hull of $\Lambda$. Now suppose that $\Phi(x) \geq 0$. This implies $\varphi(x) \geq 0$ for all $\varphi \in \Lambda$. As $x$ acts as a weak-* continuous functional on $X^{*}$, we get $\varphi(x) \geq 0$ for all $\varphi \in \Sigma$, hence for all $\varphi \in K^{*}$. Thus, $x \in K$, which shows that $\Phi$ is bipositive.

As Kadison [11, Section 4] pointed out, the map $\Phi$ can be used to prove Kakutani's representation theorem for Banach lattices, which says that each Banach lattice with an order unit is lattice isomorphic to $C(\Omega)$, where $\Omega$ is a compact Hausdorff space. In fact, he showed that $\Phi(X)=C(\bar{\Lambda})$ and $\Lambda=\bar{\Lambda}$. Of course, if $(X, K)$ is merely an Archimedean partially ordered vector space with an order unit we cannot expect $\Phi(X)$ to be norm dense in $C(\bar{\Lambda})$, but the following is true. Denote by $L(X, K)$ the Riesz subspace generated by $\Phi(X)$ inside $C(\bar{\Lambda})$; so,

$$
L(X, K)=\left\{\bigvee_{j=1}^{m} f_{j}+\bigwedge_{j=1}^{n} g_{j}: f_{1}, \ldots, f_{m}, g_{1}, \ldots, g_{n} \in \Phi(X)\right\} .
$$

Proposition 2. If $(X, K)$ is an Archimedean partially ordered vector space with an order unit, then $L(X, K)$ is norm dense in $C(\bar{\Lambda})$.

Proof. Immediate consequence of the Stone-Weierstrass theorem.

In the connection with the Riesz completion we need to consider the notion of order denseness. A linear subspace $D$ of a Riesz space $Y$ is order dense if for each $h \in Y$ we have that $h$ is the greatest lower bound of the set $\{g \in D: g \geq h\}$ in $Y$, that is,

$$
h=\inf \{g \in D: g \geq h\} .
$$

The concept of order denseness originated in $[3$, p. 360] and is essential in the definition of the Riesz-completion. A Riesz-completion of a partially ordered 
vector space $(X, K)$ is a Riesz space $Y$ for which there exists a bipositive linear map $i: X \rightarrow Y$ such that $i(X)$ is order dense in $Y$ and no proper Riesz subspace of $Y$ contains $i(X)$. It will become evident later, see also [9, Remark 3.2], that $Y$ is unique up to isomorphism, and hence we may speak of the Riesz-completion of $(X, K)$, which we denote by $(Y, i)$. A partially ordered vector space $(X, K)$ is called pre-Riesz if it has a Riesz completion. It was shown in [9, Chapter 1] that, among other spaces, every Archimedean partially ordered vector space $(X, K)$ with an order unit is pre-Riesz, but as mentioned earlier the construction of the Riesz completion is rather elaborate.

In the setting of the functional representation of Theorem 1, the Riesz subspace $L(X, K)$ in $C(\bar{\Lambda})$ is the Riesz completion of $(X, K)$ if $\Phi(X)$ is order dense in $C(\bar{\Lambda})$. But is $\Phi(X)$ always order dense in $C(\bar{\Lambda})$ ? We shall show that this question has an affirmative answer.

It is worth pointing out that if $X$ is a Riesz space and Theorem 1 is applied to $X$, then $\Phi(X)$ is a Riesz subspace of $C(\bar{\Lambda})$ and the lattice structure of $\Phi(X)$ is the induced one from $C(\bar{\Lambda})$. Thus, by applying Proposition 2 one reproduces Kakutani's famous theorem [13, Theorem 2] on representation of abstract $M$ spaces.

Theorem 3 (Kakutani). Every Archimedean Riesz space with an order unit is isometric and as a Riesz space isomorphic to a norm dense Riesz subspace of a space of continuous functions $C(\Omega)$, where $\Omega$ is a compact Hausdorff space.

\section{Functional representations and Riesz* homo- morphisms}

Let $(X, K)$ be an Archimedean partially ordered vector space with an order unit $u \in K$ and let $\Omega$ be a compact Hausdorff space and $\sigma: X \rightarrow C(\Omega)$ be a map. $(\sigma, \Omega)$ is called a functional representation of $(X, K)$ if $\sigma$ is linear, bipositive, maps $u$ to the constant 1 function (which implies that $\sigma$ is an isometry with respect to the order unit norm) and the image of $\sigma$ separates the points of $\Omega$, meaning that for each $\omega_{1} \neq \omega_{2}$ in $\Omega$ there exists $x \in X$ such that $\sigma(x)\left(\omega_{1}\right) \neq$ $\sigma(x)\left(\omega_{2}\right)$, see $[2$, p. 387]. Due to Theorem $1,(\Phi, \bar{\Lambda})$ is a functional representation of $(X, K)$ as $\Phi(X)$ separates the points of $\bar{\Lambda}$.

Let $\left(\sigma_{1}, \Omega_{1}\right)$ and $\left(\sigma_{2}, \Omega_{2}\right)$ be two functional representations of $X$. We say that $\left(\sigma_{1}, \Omega_{1}\right)$ is smaller than $\left(\sigma_{2}, \Omega_{2}\right)$ if there exists a homeomorphism $\alpha$ from $\Omega_{1}$ onto a subset of $\Omega_{2}$ such that for all $x \in X$ and all $\omega \in \Omega_{1}$ we have $\sigma_{1}(x)(\omega)=$ $\sigma_{2}(x)(\alpha(\omega))$. In a context of $C^{*}$-algebras Kadison [11] and Alfsen [2, Proposition $2]$ showed that $(\Phi, \bar{\Lambda})$ is the smallest functional representation of $X$. In this section we follow a different approach, which results in the following theorem.

Theorem 4. If $(X, K)$ is an Archimedean partially ordered vector space with an order unit $u$ and $(\sigma, \Omega)$ is a functional representation of $(X, K)$ such that $\sigma(X)$ is order dense in $C(\Omega)$, then $(\Phi, \bar{\Lambda})$ is smaller than $(\sigma, \Omega)$.

To begin we need to recall definitions and results of [9, Chapter 1]. Let $(X, K)$ be an Archimedean partially ordered vector space. For $A \subseteq X$ denote $A^{u}=\{x \in X: u \geq a$ for all $a \in A\}$ and $A^{l}=\{x \in X: x \leq a$ for all $a \in A\}$. 
A linear functional $\varphi: X \rightarrow \mathbb{R}$ is called a Riesz homomorphism if

$$
\inf \left\{\varphi(x): x \in\left\{a_{1}, \ldots, a_{m}\right\}^{u}\right\}=\bigvee_{k=1}^{m} \varphi\left(a_{k}\right)
$$

for all $a_{1}, \ldots, a_{m} \in X$ and $m \in \mathbb{N}$. It is said to be a Riesz* homomorphism if

$$
\sup \left\{\varphi(x): x \in\left\{a_{1}, \ldots, a_{m}\right\}^{u l}\right\}=\bigvee_{k=1}^{m} \varphi\left(a_{k}\right)
$$

for all $a_{1}, \ldots, a_{m} \in X$ and $m \in \mathbb{N}$. Every Riesz homomorphism is a Riesz* homomorphism and in a Riesz space the two notions coincide. The notion of a Riesz* homomorphism has been introduced by van Haandel [9, Definition 5.1, Corollary 5.4(iv)].

For the reader's convenience, we include a proof of the next theorem.

Theorem 5. [9, Theorem 5.6] Let $(X, K)$ be an Archimedean directed partially ordered vector space with Riesz completion $(Y, i)$. For a linear functional $\varphi: X \rightarrow \mathbb{R}$ the following statements are equivalent:

(i) $\varphi$ is a Riesz* homomorphism.

(ii) There exists a unique Riesz homomorphism $\psi: Y \rightarrow \mathbb{R}$ with $\psi \circ i=\varphi$.

Proof. To show that (i) implies (ii), suppose that $\bigvee_{k=1}^{m} i\left(a_{k}\right)=\bigvee_{j=1}^{n} i\left(b_{j}\right)$ for $a_{1}, \ldots, a_{m} \in X, b_{1}, \ldots, b_{n} \in X$. Using the bipositivity of $i$ we see that $x \in X$ satisfies $x \geq a_{k}$ for $k=1, \ldots, m$ if and only if $i(x) \geq \bigvee_{k=1}^{m} i\left(a_{k}\right)=\bigvee_{j=1}^{n} i\left(b_{j}\right)$, which is equivalent to $x \geq b_{j}$ for $j=1, \ldots, n$. Thus,

$$
\bigvee_{k=1}^{m} \varphi\left(a_{k}\right)=\sup \left\{\varphi(x): x \in\left\{a_{1}, \ldots, a_{n}\right\}^{u l}\right\}=\bigvee_{j=1}^{n} \varphi\left(b_{j}\right)
$$

Next, let $y \in Y$. There are $a_{1}, \ldots, a_{m}, b_{1}, \ldots, b_{n} \in X$ such that $y=$ $\bigvee_{k=1}^{m} i\left(a_{k}\right)-\bigvee_{j=1}^{n} i\left(b_{j}\right)$. Suppose also $y=\bigvee_{k=1}^{p} i\left(c_{k}\right)-\bigvee_{j=1}^{q} i\left(d_{j}\right)$ for some $c_{1}, \ldots, c_{p}, d_{1}, \ldots, d_{q} \in X$. Then $\bigvee_{k=1}^{m} i\left(a_{k}\right)+\bigvee_{j=1}^{q} i\left(d_{j}\right)=\bigvee_{k=1}^{p} i\left(c_{k}\right)+\bigvee_{j}^{n} i\left(b_{j}\right)$, $\mathrm{SO}$

$$
\begin{aligned}
\bigvee_{k=1}^{m} \bigvee_{j=1}^{q} i\left(a_{k}+d_{j}\right) & =\bigvee_{k=1}^{m}\left(i\left(a_{k}\right)+\bigvee_{j=1}^{q} i\left(d_{j}\right)\right) \\
& =\bigvee_{k=1}^{m} i\left(a_{k}\right)+\bigvee_{j=1}^{q} i\left(d_{j}\right)=\bigvee_{k=1}^{p} \bigvee_{j=1}^{n} i\left(c_{k}+b_{j}\right)
\end{aligned}
$$

Therefore $\bigvee_{k=1}^{m} \bigvee_{j=1}^{q} \varphi\left(a_{k}+d_{j}\right)=\bigvee_{k=1}^{p} \bigvee_{j=1}^{n} \varphi\left(c_{k}+b_{j}\right)$, hence $\bigvee_{k=1}^{m} \varphi\left(a_{k}\right)-$ $\bigvee_{j=1}^{n} \varphi\left(b_{j}\right)=\bigvee_{k=1}^{p} \varphi\left(c_{k}\right)-\bigvee_{j=1}^{q} \varphi\left(d_{j}\right)$. It follows that $\psi: Y \rightarrow \mathbb{R}$ is well defined by setting

$$
\psi(y)=\bigvee_{k=1}^{m} \varphi\left(a_{k}\right)-\bigvee_{j=1}^{n} \varphi\left(b_{j}\right)
$$

We show that $\psi$ is a Riesz homomorphism. For $y, z \in Y$ choose $a_{k}, b_{j}, c_{m}, d_{n} \in Y$ and $a_{k}^{\prime}, b_{j}^{\prime}, c_{m}^{\prime}, d_{n}^{\prime} \in X$ such that $a_{k}=i\left(a_{k}^{\prime}\right), b_{j}=i\left(b_{j}^{\prime}\right), c_{m}=i\left(c_{m}^{\prime}\right), d_{n}=i\left(d_{n}^{\prime}\right)$, 
and $y=\bigvee_{k} a_{k}-\bigvee_{j} b_{j}$ and $z=\bigvee_{m} c_{m}-\bigvee_{n} d_{n}$. Then

$$
\begin{aligned}
y \vee z & =\left(\bigvee_{k} a_{k}+\bigvee_{n} d_{n}\right) \vee\left(\bigvee_{m} c_{m}+\bigvee_{j} b_{j}\right)-\left(\bigvee_{j} b_{j}+\bigvee_{n} d_{n}\right) \\
& =\bigvee_{k} \bigvee_{n}\left(a_{k}+d_{n}\right) \vee \bigvee_{m} \bigvee_{j}\left(c_{m}+b_{j}\right)-\bigvee_{j} \bigvee_{n}\left(b_{j}+d_{n}\right),
\end{aligned}
$$

so

$$
\begin{aligned}
\psi(y \vee z)=\bigvee_{k} \bigvee_{n}\left(\varphi\left(a_{k}^{\prime}\right)+\varphi\left(d_{n}^{\prime}\right)\right) \vee \bigvee_{m} \bigvee_{j}\left(\varphi\left(c_{m}^{\prime}\right)-\varphi\left(b_{j}^{\prime}\right)\right) \\
-\bigvee_{n} \bigvee_{j}\left(\varphi\left(b_{j}^{\prime}\right)+\varphi\left(d_{n}^{\prime}\right)\right) \\
=\left(\bigvee_{k} \varphi\left(a_{k}^{\prime}\right)-\bigvee_{j} \varphi\left(b_{j}^{\prime}\right)\right) \vee\left(\bigvee_{m} \varphi\left(c_{m}^{\prime}\right)-\bigvee_{n} \varphi\left(d_{n}^{\prime}\right)\right) \\
=\psi\left(\bigvee_{k} a_{k}-\bigvee_{j} b_{j}\right) \vee \psi\left(\bigvee_{m} c_{m}-\bigvee_{n} d_{n}\right)=\psi(y) \vee \psi(z) .
\end{aligned}
$$

Further, $y+z=\bigvee_{k} \bigvee_{m}\left(a_{k}+c_{m}\right)-\bigvee_{j} \bigvee_{n}\left(b_{j}+d_{n}\right)$, so

$$
\psi(y+z)=\bigvee_{n} \bigvee_{m}\left(\varphi\left(a_{k}^{\prime}\right)+\varphi\left(c_{m}^{\prime}\right)\right)-\bigvee_{j} \bigvee_{n}\left(\varphi\left(b_{j}^{\prime}\right)+\varphi\left(d_{n}^{\prime}\right)\right)=\psi(y)+\psi(z)
$$

In a similar fashion we infer $\psi(\alpha y)=\alpha \psi(y)$ for $\alpha \in \mathbb{R}^{+}$, and $\psi(-y)=-\psi(y)$.

Finally, if $\eta: Y \rightarrow \mathbb{R}$ is a Riesz homomorphism with $\eta \circ i=\varphi$, then

$$
\eta(y)=\bigvee_{k} \eta\left(a_{k}\right)-\bigvee_{j} \eta\left(b_{j}\right)=\bigvee_{k} \varphi\left(a_{k}^{\prime}\right)-\bigvee_{j} \varphi\left(b_{j}^{\prime}\right)=\psi(y) .
$$

Hence $\psi$ is the unique Riesz homomorphism from $Y$ into $\mathbb{R}$ with $\psi \circ i=\varphi$.

To show that (ii) implies (i), let $a_{1}, \ldots, a_{m} \in X$. If $x \in\left\{a_{1}, \ldots, a_{n}\right\}^{u l}$, then $x \leq v$ for every $v \in\left\{a_{1}, \ldots, a_{n}\right\}^{u}$, so $i(x) \leq i(v)$ for every $v \in X$ with $i(v) \geq i\left(a_{k}\right)$ for all $k$. By order denseness of $i(X)$ in $Y$,

$$
\bigvee_{k=1}^{m} i\left(a_{k}\right)=\inf \left\{i(v): v \in\left\{a_{1}, \ldots, a_{n}\right\}^{u}\right\} \geq i(x),
$$

so that

$$
\varphi(x)=\psi(i(x)) \leq \bigvee_{k=1}^{m} \psi\left(i\left(a_{k}\right)\right)=\bigvee_{k=1}^{m} \varphi\left(a_{k}\right)
$$

Since $a_{k} \in\left\{a_{1}, \ldots, a_{m}\right\}^{u l}$ for each $k$, we obtain that $\varphi$ is a Riesz* homomorphism.

Let $(X, K)$ be an Archimedean partially ordered vector space with an order unit $u$. The first part of the following proposition is a well-known theorem by Hayes [10, Theorem 1.8.1]. We shall only prove the second part, which can also be found in the work by van Haandel [9, Theorem 5.10(iii)].

Proposition 6. For each $\varphi \in \Sigma$ we have 
(i) $\varphi \in \Lambda$ if and only if $\varphi$ is a Riesz homomorphism; and

(ii) $\varphi \in \bar{\Lambda}$ if and only if $\varphi$ is a Riesz* homomorphism.

Proof. Let $\varphi \in \bar{\Lambda}$. Choose a net of Riesz homomorphisms $\varphi_{i}$ that converges to $\varphi$ in the weak-* topology. Let $A=\left\{a_{1}, \ldots, a_{m}\right\}$ be a non-empty finite subset of $X$ and let $x \in A^{u l}$. Then $x \leq v$ for every upper bound $v$ of $A$, so

$$
\varphi_{i}(x) \leq \varphi_{i}\left(a_{1}\right) \vee \cdots \vee \varphi_{i}\left(a_{m}\right) \text { for all } i .
$$

Then $\varphi(x) \leq \varphi\left(a_{1}\right) \vee \cdots \vee \varphi\left(a_{m}\right)$ and $\varphi$ is a Riesz* homomorphism.

To prove the converse, let $(Y, i)$ be the Riesz completion of $(X, K)$ and let $\mathcal{A}$ denote the set of Riesz homomorphisms $\psi: Y \rightarrow \mathbb{R}$ with $\psi(i(u))=1$. Due to (i), $\mathcal{A}$ is the set of all extremal points of

$$
\Sigma_{Y}=\{\psi: Y \rightarrow \mathbb{R}: \psi \text { positive and } \psi(i(u))=1\}
$$

and $\mathcal{A}$ is easily seen to be weak-* closed. Hence $\mathcal{A}$ is weak-* compact. Let $\mathcal{C}$ be the set of Riesz* homomorphisms $\varphi: X \rightarrow \mathbb{R}$ with $\varphi(u)=1$. According to Theorem 5, the map $\pi: \mathcal{A} \rightarrow \mathcal{C}$ given by $\pi(\psi)=\psi \circ i$ is a bijection. More than that, $\pi$ is a homeomorphism with respect to the weak-* topologies. So $\bar{\Lambda}$ is compact as a closed subset of $\mathcal{C}=\pi(\mathcal{A})$.

If $z \in Y$ is such that $\psi(z) \geq 0$ for all $\psi \in \pi^{-1}(\Lambda)$, then for each $x \in X$ with $i(x) \geq z$ we have $\psi(i(x)) \geq 0$, so $\varphi(x) \geq 0$ for all $\varphi \in \Lambda$, hence $x \geq 0$. By order denseness of $i(X)$ in $Y$ it follows that $z \geq 0$.

Next we show that the closed convex hull $\overline{c o} \pi^{-1}(\bar{\Lambda})$ of $\pi^{-1}(\bar{\Lambda})$ equals $\Sigma_{Y}$. Suppose not. Choose $\psi_{0} \in \Sigma_{Y} \backslash \overline{\mathrm{co}} \pi^{-1}(\bar{\Lambda})$. By Hahn-Banach, there exist $y \in Y$ and $\alpha \in \mathbb{R}$ such that $\psi_{0}(y)<\alpha$ and $\psi(y) \geq \alpha$ for all $\psi \in \overline{\operatorname{co}} \pi^{-1}(\bar{\Lambda})$. Take $z=y-\alpha i(u)$. Then $\psi_{0}(z)<0$ and $\psi(z) \geq 0$ for all $\psi \in \overline{\operatorname{co}} \pi^{-1}(\bar{\Lambda})$, so $\psi(z) \geq 0$ for all $\psi \in \pi^{-1}(\Lambda)$. By the previous step, we obtain $z \geq 0$, which contradicts $\psi_{0}(z)<0$. Hence $\overline{\mathrm{co}} \pi^{-1}(\bar{\Lambda})=\Sigma_{Y}$.

According to [4, Lemma V.8.5], the extremal points of $\overline{\mathrm{co}} \pi^{-1}(\bar{\Lambda})$ are contained in $\pi^{-1}(\bar{\Lambda})$, so that $\mathcal{A} \subseteq \pi^{-1}(\bar{\Lambda})$. Hence $\mathcal{C}=\pi(\mathcal{A}) \subseteq \bar{\Lambda}$.

The following two lemmas provide the link between the Riesz completion of an Archimedean partially ordered vector space with order unit and its smallest functional representation.

Lemma 7. Let $(\sigma, \Omega)$ be a functional representation of $X$ such that $\sigma(X)$ is order dense in $C(\Omega)$. For every $\varphi \in \bar{\Lambda}$ there exists a unique $\omega \in \Omega$ such that for all $x \in X$ we have $\varphi(x)=\sigma(x)(\omega)$.

Proof. Let $R$ be the Riesz subspace of $C(\Omega)$ generated by $\sigma(X)$. Then $\sigma(X)$ is order dense in $R$, so $R$ is the Riesz completion of $X$. Let $\varphi \in \bar{\Lambda}$. Due to Proposition $6 \varphi$ is a Riesz* homomorphism on $X$. Since $\sigma$ is bipositive (and hence injective), $\varphi \circ \sigma^{-1}$ is a Riesz* homomorphism on $\sigma(X)$. Due to Theorem 5 there exists a unique Riesz homomorphism $\psi: R \rightarrow \mathbb{R}$ extending $\varphi \circ \sigma^{-1}$. As $R$ contains the constant 1 function and $\psi$ is positive, $\psi$ is continuous. By the Stone-Weierstrass theorem the subspace $R$ is dense in $C(\Omega)$, so that $\psi$ extends to a linear functional $\bar{\psi}: C(\Omega) \rightarrow \mathbb{R}$, which is a Riesz homomorphism since the lattice operations are continuous. (Alternatively, one could directly use the Lipecki-Luxemburg-Schep extension theorem, see e.g. [1, Theorem 7.17], to 
obtain $\bar{\psi}$.) Thus there exists $\omega \in \Omega$ such that for all $f \in C(\Omega)$ we have that $\bar{\psi}(f)=f(\omega)[1$, Theorem 7.21]. In particular, $\varphi(x)=\psi(\sigma(x))=\sigma(x)(\omega)$ for all $x \in X$. Finally note that the uniqueness of $\omega$ follows since $\sigma(X)$ separates the points of $\Omega$.

By Lemma 7 there exists a unique map $\alpha: \bar{\Lambda} \rightarrow \Omega$ such that for all $\varphi \in \bar{\Lambda}$ and for all $x \in X$ we have

$$
\varphi(x)=\sigma(x)(\alpha(\varphi)) .
$$

If $\varphi_{1}, \varphi_{2} \in \bar{\Lambda}$ are such that $\alpha\left(\varphi_{1}\right)=\alpha\left(\varphi_{2}\right)$, then for all $x \in X$ we have that $\varphi_{1}(x)=\varphi_{2}(x)$, so $\varphi_{1}=\varphi_{2}$. Hence, $\alpha$ is injective. We define $\Gamma=\alpha(\bar{\Lambda})$ and prove the following lemma.

Lemma 8. The bijection $\alpha: \bar{\Lambda} \rightarrow \Gamma$ is a homeomorphism.

Proof. Let $\left(\varphi_{i}\right)_{i \in I}$ be a net in $\bar{\Lambda}$ and let $\varphi \in \bar{\Lambda}$ be such that $\varphi_{i} \rightarrow \varphi$. Then for all $x \in X$ we have $\varphi_{i}(x) \rightarrow \varphi(x)$, so $\sigma(x)\left(\alpha\left(\varphi_{i}\right)\right) \rightarrow \sigma(x)(\alpha(\varphi))$. Let $R$ be the Riesz subspace of $C(\Omega)$ generated by $\sigma(X)$. As the lattice operations in $C(\Omega)$ are pointwise, we find for each $g \in R$ that $g\left(\alpha\left(\varphi_{i}\right)\right) \rightarrow g(\alpha(\varphi))$. Suppose that the net $\left(\alpha\left(\varphi_{i}\right)\right)_{i \in I}$ does not converge to $\alpha(\varphi)$. Then there is an open set $U \subseteq \Omega$ with $\alpha(\varphi) \in U$ such that for every $i \in I$ there is $j \in I$ with $j \geq i$ and $\alpha\left(\varphi_{j}\right) \notin U$. According to Urysohn's Lemma there exists a function $f \in C(\Omega)$ with $0 \leq f \leq 1, f(\alpha(\varphi))=1$ and $f(\omega)=0$ for all $\omega \in \Omega \backslash U$. Since $R$ is norm dense in $C(\Omega)$, there exists $g \in R$ such that $g(\alpha(\varphi))>1 / 2$ and $g(\omega)<1 / 2$ for all $\omega \in \Omega \backslash U$. But then for every $i \in I$ there exists $j \in I$ with $j \geq i$ such that $g\left(\alpha\left(\varphi_{j}\right)\right)<1 / 2$, which contradicts $g\left(\alpha\left(\varphi_{i}\right)\right) \rightarrow g(\alpha(\varphi))$. Thus $\alpha$ is continuous.

It remains to show that $\alpha^{-1}$ is continuous. Let $\left(\omega_{i}\right)_{i \in I}$ be a net in $\Gamma$ and let $\omega \in \Gamma$ be such that $\omega_{i} \rightarrow \omega$. Denote $\varphi_{i}=\alpha^{-1}\left(\omega_{i}\right)$ for all $i \in I$ and $\varphi=\alpha^{-1}(\omega)$, so $\alpha\left(\varphi_{i}\right) \rightarrow \alpha(\varphi)$. We obtain for every $x \in X$ that $\varphi_{i}(x)=\sigma(x)\left(\alpha\left(\varphi_{i}\right)\right) \rightarrow$ $\sigma(x) \alpha(\varphi)=\varphi(x)$. Consequently, $\varphi_{i} \rightarrow \varphi$, so $\alpha^{-1}$ is continuous.

Remark that Theorem 4 is a direct consequence of Lemmas 7 and 8.

\section{A representation of the Riesz completion}

In this section we show that if $(X, K)$ is an Archimedean partially ordered vector space with an order unit $u$, then $\Phi(X)$ is order dense in $C(\bar{\Lambda})$, and hence the vector lattice $L(X, K)$ generated by $\Phi(X)$ in $C(\bar{\Lambda})$ is a representation of the Riesz completion. Before we start the proof it is convenient to isolate the following technical lemma concerning order denseness, which plays a role in our construction that is similar to [9, Theorem 5.7] in van Haandel's work.

Lemma 9. If $Z$ is an Archimedean Riesz space with an order unit $u, Y$ is a norm dense Riesz subspace of $Z$, and $X$ is an order dense subspace of $Y$ containing $u$, then $X$ is order dense in $Z$.

Proof. Suppose that there exists an $h \in Z$ such that $h$ is not the greatest lower bound of $H=\{f \in X: f \geq h\}$. Clearly $h$ is a lower bound of $H$, so then there exists a $g_{0} \in Z$ with $g_{0} \leq f$ for all $f \in H$ but $g_{0} \not \leq h$. Let $g=g_{0} \vee h$. Then $g \geq h, g \neq h$, and $g$ is a lower bound of $H$, so $f \in X$ and $f \geq h$ implies $f \geq g$. 
Take $\delta>0$ such that $4 \delta<\|g-h\|$. By norm denseness there exist $y_{1}, y_{2} \in Y$ with $\left\|y_{1}-h\right\|<\delta$ and $\left\|y_{2}-g\right\|<\delta$.

The remaining part of the proof makes use of the fact that $\|a-b\|<\varepsilon$ implies $b-\varepsilon u \leq a \leq b+\varepsilon u$, which follows directly from the definition of the order unit norm. If $f \in X$ and $f \geq y_{1}$, then $f+\delta u \in X$ and $f+\delta u \geq y_{1}+\delta u \geq h$, so $f+\delta u \geq g$ and therefore $f \geq g-\delta u \geq y_{2}-2 \delta u$. Due to the order denseness of $X$ in $Y$ we obtain

$$
y_{1}=\inf \left\{f \in X: f \geq y_{1}\right\} \geq \inf \left\{f \in X: f \geq y_{2}-2 \delta u\right\}=y_{2}-2 \delta u,
$$

so that $h \geq y_{1}-\delta u \geq y_{2}-3 \delta u \geq g-4 \delta u$. Then $0 \leq g-h \leq 4 \delta u$, so $\|g-h\| \leq 4 \delta$, which contradicts $4 \delta<\|g-h\|$. Thus $h=\inf H$ and $X$ is order dense in $Z$.

Let $(X, K)$ be an Archimedean partially ordered vector space with an order unit $u \in K$. Denote the Riesz completion of $(X, K)$ by $(Y, i)$, and recall that $i(u)$ is an order unit in $Y$, since $i(X)$ is majorizing in $Y$. Moreover, $Y$ is Archimedean. Due to Kakutani's Theorem 3 there exists a compact Hausdorff space $\Omega$ and a linear injection $\pi: Y \rightarrow C(\Omega)$ such that $\pi: Y \rightarrow \pi(Y)$ is a lattice isomorphism which maps $i(u)$ to the constant 1 function in $C(\Omega)$, and $\pi(Y)$ is norm dense in $\left(C(\Omega),\|\cdot\|_{\infty}\right)$. Using this notation we now formulate the following theorem.

Theorem 10. If $(X, K)$ is an Archimedean partially ordered vector space with an order unit $u$, then

(i) the compact Hausdorff spaces $\bar{\Lambda}$ and $\Omega$ are homeomorphic, and

(ii) $\Phi(X)$ is order dense in $C(\bar{\Lambda})$.

Proof. First we show that $(\pi \circ i, \Omega)$ is a functional representation of $X$. The map $\pi \circ i: X \rightarrow C(\Omega)$ is linear bipositive and maps $u$ to the constant 1 function in $C(\Omega)$ (hence it is isometric). It remains to show that $\pi(i(X))$ separates points in $\Omega$. Suppose not, then there are $\omega_{1}, \omega_{2} \in \Omega, \omega_{1} \neq \omega_{2}$, such that $f\left(\omega_{1}\right)=f\left(\omega_{2}\right)$ for all $f \in \pi(i(X))$. The space $\pi(Y)$ is the lattice generated by $\pi(i(X))$, since $\pi$ is a lattice isomorphism and $i(X)$ generates $Y$ as a vector lattice. Hence, $f\left(\omega_{1}\right)=f\left(\omega_{2}\right)$ for all $f \in \pi(Y)$. Due to Theorem $3 \pi(Y)$ is norm dense in $C(\Omega)$, so $C(\Omega)$ does not separate the points of $\Omega$, which is a contradiction.

We apply Lemma 9 (with $Z=C(\Omega)$ ) and obtain that $\pi(i(X))$ is order dense in $C(\Omega)$. Due to Theorem 4 we have that $(\Phi, \bar{\Lambda})$ is smaller than $(\pi \circ i, \Omega)$. So, there exists $\Gamma \subseteq \Omega$ and a homeomorphism $\alpha: \bar{\Lambda} \rightarrow \Gamma$ such that for all $x \in X$ and all $\varphi \in \bar{\Lambda}$ we have $\Phi(x)(\varphi)=\pi(i(x))(\alpha(\varphi))$.

We show that $\Gamma=\Omega$. Fix $\omega \in \Omega$. Define $\psi: C(\Omega) \rightarrow \mathbb{R}, f \mapsto f(\omega)$. Then $\psi$ is a Riesz homomorphism and $\psi(1)=1$. The restriction of $\psi$ to $\pi(Y)$ is a Riesz homomorphism as well. Since $\pi$ is bipositive, $(\pi(Y), \pi \circ i)$ is a Riesz completion of $X$. Due to Theorem 5 the map $\varphi: X \rightarrow \mathbb{R}$ given by $\varphi:=\psi \circ \pi \circ i$ is a Riesz* homomorphism with $\varphi(u)=1$. According to Proposition 6 we obtain $\varphi \in \bar{\Lambda}$. For every $x \in X$ we have

$$
\pi(i(x))(\alpha(\varphi))=\Phi(x)(\varphi)=\varphi(x)=\psi(\pi(i(x)))=\pi(i(x))(\omega) .
$$

Since $\pi(i(X))$ separates the points of $\Omega$, it follows that $\alpha(\varphi)=\omega$. 
Finally we show that $\Phi(X)$ is order dense in $C(\bar{\Lambda})$. Define $A: C(\Omega) \rightarrow C(\bar{\Lambda})$, $f \mapsto f \circ \alpha$. Then $A$ is an isometric Riesz isomorphism as $\alpha$ is a homeomorphism. Hence $A(\pi(i(X)))$ is order dense in $C(\bar{\Lambda})$. For each $x \in X$ and $\varphi \in \bar{\Lambda}$ we have

$$
A(\pi(i(x)))(\varphi)=\pi(i(x))(\alpha(\varphi))=\Phi(x)(\varphi),
$$

so $A(\pi(i(x)))=\Phi(x)$. We obtain $A(\pi(i(X)))=\Phi(X)$, therefore $\Phi(X)$ is order dense in $C(\bar{\Lambda})$.

\section{Computing the Riesz completion}

In this section we show how the functional representation can be used to compute the Riesz completion for a variety of interesting examples.

Lorentz cones Let $H$ be a (possibly infinite dimensional) Hilbert space with inner product $\langle\cdot, \cdot\rangle$ and define $X=\mathbb{R} \times H$. The Lorentz cone in $X$ is given by

$$
L_{H}=\left\{(r, u) \in \mathbb{R} \times H: r^{2}-\langle u, u\rangle \geq 0 \text { and } r \geq 0\right\} .
$$

In case $H=\mathbb{R}^{2}$ we get the familiar ice-cream cone. On $X$ we consider the inner product $\langle x \mid y\rangle=r s+\langle u, v\rangle$, where $x=(r, u)$ and $y=(s, v)$, which turns $X$ into a Hilbert space. With respect to this inner product $L_{H}$ is self-dual. To show this consider $y=(s, v) \in L_{H}$. For each $x=(r, u) \in L_{H}$ we have, by Cauchy-Schwartz, that

$$
\langle x \mid y\rangle=r s+\langle u, v\rangle \geq r s-\|u\|\|v\| \geq 0 .
$$

Thus, $y \in L_{H}^{*}$. (Here $L_{H}^{*}$ denotes the dual of $L_{H}$ with respect to the Hilbert norm.) Conversely, if $y=(s, v) \in L_{H}^{*}$, then $s \geq 0$. If $v=0$, then clearly $y \in L_{H}$. If $v \neq 0$, then consider $x=(r, u)$, where $r=\|v\|$ and $u=-v$. As $r^{2}-\langle u, u\rangle=0, x \in L_{H}$, and hence $\langle x \mid y\rangle \geq 0$. This implies that

$$
\langle x \mid y\rangle=r s-\langle v, v\rangle=\|v\| s-\|v\|^{2} \geq 0,
$$

so that $s-\|v\| \geq 0$, which shows that $y \in L_{H}$.

Note that $L_{H}$ has non-empty interior. In fact, it contains the point $e=(1,0)$. Indeed, $\langle e-(r, v) \mid e-(r, v)\rangle<1 / 4$ implies $(1-r)^{2}+\|v\|^{2}<1 / 4$, which gives $1 / 2<r<3 / 2$ and $\|v\|<1 / 2$. Therefore, $r^{2}-\|v\|^{2}>0$ and hence $(r, v) \in L_{H}$. The point $e$ is an order unit for $L_{H}$.

As $L_{H}$ has non-empty interior, it follows from [16, Corollary 2.17] that every positive functional on $L_{H}$ is continuous under the Hilbert norm on $X$. This implies that $K^{*}=L_{H}^{*}=L_{H}$, so that $\Sigma=\left\{(r, u) \in L_{H}: r=1\right\}$. Clearly the extreme points of $\Sigma$ are contained in $\left\{(1, u) \in L_{H}:\|u\|=1\right\}$.

We claim that each point $z=(1, w)$ with $\|w\|=1$ is extreme. To prove this let $x=(1, u)$ and $y=(1, v)$ be such that $u \neq v,\|u\| \leq 1$ and $\|v\| \leq 1$. For the sake of contradiction suppose that $z=\lambda x+(1-\lambda) y$ with $0<\lambda<1$. As

$$
1-\|\lambda u+(1-\lambda) v\|^{2}>1-(\lambda\|u\|+(1-\lambda)\|v\|)^{2} \geq 1-(\lambda+(1-\lambda))^{2}=0,
$$

we find that $\|w\|<1$, which is a contradiction. Thus, the set of extreme points is $\Lambda=\left\{(1, u) \in L_{H}:\|u\|=1\right\}$. By applying Theorem 10 we conclude that $\Phi(X)$ is order dense in $C(\bar{\Lambda})$ and the Riesz completion of $\left(X, L_{H}\right)$ is $L\left(X, L_{H}\right)$. If $H=\mathbb{R}^{n}$ we write $L_{n}$ to denote the Lorentz cone. In that case the set $\bar{\Lambda}=\Lambda$ can be identified with the $(n-1)$-sphere, $S^{n-1}$. 
Positive semi-definite matrices Let $V$ be the vector space of $n \times n$ symmetric matrices equipped with the inner-product $\langle A, B\rangle=\operatorname{tr}(A B)$. Let $\operatorname{Pos}_{n}$ be the cone of positive semi-definite matrices in $V$. It is well-known [5] that $\mathrm{Pos}_{n}$ is self-dual in $V$ and that the identity matrix $I$ is an order unit. Thus,

$$
\Sigma=\left\{A \in \operatorname{Pos}_{n}: \operatorname{tr}(A)=1\right\} .
$$

The following lemma characterises the extreme points of $\Lambda$.

Lemma 11. The set $\Lambda$ consisting of the extreme points of $\Sigma$ is equal to the closed set $\Delta$ consisting of those $A \in \Sigma$ that have exactly one non-zero eigenvalue, which is equal to 1 .

Proof. First note that if $A \in \Sigma$, then by the spectral theorem there exists an orthogonal matrix $S$ such that

$$
A=S^{T} \operatorname{Diag}\left(\lambda_{1}, \ldots, \lambda_{n}\right) S=\sum_{i} \lambda_{i} S^{T} \operatorname{Diag}\left(e_{i}\right) S,
$$

where $\lambda_{n} \geq \lambda_{n-1} \geq \ldots \geq \lambda_{1} \geq 0$ are the eigenvalues of $A, \sum_{i} \lambda_{i}=1$, and $e_{i}$ is the $i$-th unit vector. Thus, $\Lambda$ is contained in $\Delta$. To see that each $A \in \Delta$ is an extreme point, we remark that the group of orthogonal matrices, $O(n)$, acts transitively on $\Delta$ by conjugation, i.e., for $A, B \in \Delta$ there exists $Q \in O(n)$ such that $Q^{T} A Q=B$. For each $Q \in O(n)$ the map $A \mapsto Q^{T} A Q$ is an invertible linear map on $V$ which maps $\Sigma$ onto itself. So, it maps extreme points of $\Sigma$ to extreme points of $\Sigma$. As $\Lambda$ is a non-empty subset of $\Delta$ and $O(n)$ acts transitively on $\Delta$, it follows that each $A \in \Delta$ is an extreme point of $\Sigma$. Thus, $\Lambda=\Delta$. It is easy to see that $\Delta$ is closed.

Let $S^{n-1}$ be the $(n-1)$-sphere and consider the equivalence relation $\sim$ in $S^{n-1}$ given by $q \sim p$ if $q=p$ of $q=-p$.

Lemma 12. The map $[q] \in S^{n-1} / \sim \mapsto q q^{T} \in \Lambda$ is a bijection.

Proof. If $p, q \in S^{n-1}$ and $p p^{T}=q q^{T}$, then $p=q q^{T} p$, so $p$ is a multiple of $q$, consequently $p=q$ or $p=-q$. Hence, the map is injective.

To show that it is surjective, let $A \in \Lambda$ and $E=\operatorname{Diag}(1,0, \ldots, 0)$. By the spectral theorem there exists an orthogonal matrix $Q=\left(q_{1} q_{2} \cdots q_{n}\right)$, where $q_{1}, \ldots, q_{n}$ denote the columns of $Q$, such that $Q^{T} A Q=E$, so $A=Q E Q^{T}=$ $q_{1} q_{1}^{T}$, while $q_{1} \in S^{n-1}$.

Remark that for $q \in S^{n-1}$ the matrix $q q^{T}$ is an orthogonal projection matrix onto the span of $q$. With the identification in Lemma 12 we obtain the Riesz completion of $V$ as an order dense Riesz subspace of $C\left(S^{n-1} / \sim\right)$, with the embedding map $\Phi(A)([q])=\operatorname{tr}\left(A\left(q q^{T}\right)\right)$ for $A \in V$.

Polyhedral cones In this example we consider closed polyhedral cones $K$ in $\mathbb{R}^{n}$ which have a point $u$ in the interior. For such cones there exist $k \geq n$ linear functionals $f_{1}, \ldots, f_{k}$ with $f_{i}(u)=1$ for all $i$ such that

$$
K=\left\{x \in \mathbb{R}^{n}: f_{i}(x) \geq 0 \text { for } i=1, \ldots, k\right\},
$$

and each $f_{i}$ defines a facet of $K$, i.e., $\operatorname{dim}\left(\left\{x \in K: f_{i}(x)=0\right\}\right)=n-1$. The dual cone $K^{*}$ is also a polyhedral cone in $\mathbb{R}^{n}$. Moreover, $\Sigma=\operatorname{co}\left(f_{1}, \ldots, f_{k}\right)$ and $\Lambda=\left\{f_{1}, \ldots, f_{k}\right\}$. So it follows from Theorem 10 that $\Phi\left(\mathbb{R}^{n}\right)$ is order dense in $\left(\mathbb{R}^{k}, \mathbb{R}_{+}^{k}\right)$. It turns out that $L\left(\mathbb{R}^{n}, K\right)=\left(\mathbb{R}^{k}, \mathbb{R}_{+}^{k}\right)$ in this case. 
Proposition 13. If $K$ is a polyhedral cone with $k$ facets and non-empty interior, then $\Phi\left(\mathbb{R}^{n}\right)$ is order dense in $\left(\mathbb{R}^{k}, \mathbb{R}_{+}^{k}\right)$, and $\left(\mathbb{R}^{k}, \mathbb{R}_{+}^{k}\right)$ is the Riesz completion of $\left(\mathbb{R}^{n}, K\right)$.

Proof. It remains to show that $L\left(\mathbb{R}^{n}, K\right)=\mathbb{R}^{k}$. Fix $1 \leq l \leq k$ and consider the unit vector $y \in \mathbb{R}^{k}$ with $y_{i}=1$ if $i=l$ and $y_{i}=0$ otherwise. Observe that $f_{\ell}$ is exposed, i.e., there exists $x \in K$ such that $f_{l}(x)=0$ and $f_{i}(x)>0$ whenever $i \neq l$. Choose $\alpha=\min _{i \neq l} f_{i}(x)$ and $a=u-\alpha^{-1} x$. Then $\Phi(a) \vee 0=y$. Hence the smallest Riesz subspace of $\mathbb{R}^{k}$ containing $\Phi\left(\mathbb{R}^{n}\right)$ equals $\mathbb{R}^{k}$.

In view of Proposition 13 it is natural to ask which Archimedean partially ordered vector spaces $(X, K)$ with an order unit $u$ satisfy $L(X, K)=C(\bar{\Lambda})$. Besides polyhedral cones there exist infinite dimensional examples for which this equality holds. For instance, the equality can be shown for the space $(X, K)$ of [8, Example 2.2]. So far, however, no general characterization of such partially ordered vector spaces exists.

\section{Anti-lattices and disjointness}

Recall that a partially order vector space $(X, K)$ is an anti-lattice if $x$ and $y$ are comparable in $X$ whenever $x$ and $y$ have a greatest lower bound. This notion was introduced by Kadison [12] and indicates that there is very little lattice structure present in the partially ordered vector space. As we shall see in the next theorem the notion of an anti-lattice is closely related to the existence of non-trivial disjoint elements in $K$. Recall that two elements $x$ and $y$ in an Archimedean partially ordered vector space $(X, K)$ are disjoint, denoted $x \perp y$, if the set of all upper bounds of $\{x+y, x-y\}$ equals the set of all upper bounds of $\{x-y,-x+y\}$, see [6]. Using the Riesz completion the notion of disjointness can be translated to the familiar disjointness in a Riesz space. The advantage of the functional representation is that the Riesz completion can now be conveniently computed. If $(Y, i)$ is the Riesz completion of $(X, K)$, then $x \perp y$ if and only if $i(x) \perp i(y)$, see [6, Theorem 4.1]. Thus, it follows from Theorem 10 that $x \perp y$ in $X$ if, and only if, the functions $\Phi(x)$ and $\Phi(y)$ are disjoint in $C(\bar{\Lambda})$. We will use this insight to investigate anti-lattices by means of disjointness.

Theorem 14. Suppose that $(X, K)$ is pre-Riesz. If $x, y \in K$ and $x \perp y$, then $x \vee y$ exists and equals $x+y$. Moreover, $X$ is an anti-lattice if and only if there are no non-trivial disjoint elements in $K$.

Proof. Let $x, y \in K$ with $x \perp y$. According to [6, Theorem 4.1] this implies $i(x) \perp i(y)$ in the Riesz completion $Y$, so $i(x) \vee i(y)=i(x)+i(y)=i(x+y)$. Consequently, $i(x) \vee i(y) \in i(X)$, which implies that $x \vee y$ exists in $X$. Moreover, $x \vee y=x+y$.

Now assume there are elements $x, y \in K \backslash\{0\}$ with $x \perp y$. Then $x \vee y$ exists by the previous assertion and $x$ and $y$ are not comparable (otherwise $x \leq y$ would imply $i(x) \leq i(y)$, so $i(x) \not \perp i(y)$, which yields $x \not \perp y$, see [6]).

Assume that $X$ is not an anti-lattice, that is, there are $x, y \in X$ not comparable, but $x \vee y$ exists. The elements $x \vee y-y$ and $x \vee y-x$ are positive and nontrivial, as otherwise $x$ and $y$ would be comparable. We now show that they 
are disjoint. Remark that

$$
\begin{aligned}
i(x \vee y-y) \wedge i(x \vee y-x) & =(i(x) \vee i(y)-i(y)) \wedge(i(x) \vee i(y)-i(x)) \\
& =(i(x)-i(y))^{+} \wedge(i(x)-i(y))^{-}=0 .
\end{aligned}
$$

So, $i(x \vee y-y) \perp i(x \vee y-x)$ in $Y$, which implies $x \vee y-y \perp x \vee y-x$ in $X$.

The next example shows that there exist anti-lattices with disjoint elements. So, it may happen that an anti-lattice possesses some lattice structure.

An anti-lattice with disjoint elements Let $T=\{(\cos \vartheta, \sin \vartheta, 0,1) \in$ $\left.\mathbb{R}^{4}: 0 \leq \vartheta<2 \pi\right\}$ and let

$$
S=\operatorname{co}(\{(0,0,-1,1),(0,0,1,1)\} \cup\{\tau: \tau \in T\}) .
$$

By projecting out the last coordinate we can visualize $S$ as a subset of $\mathbb{R}^{3}$.

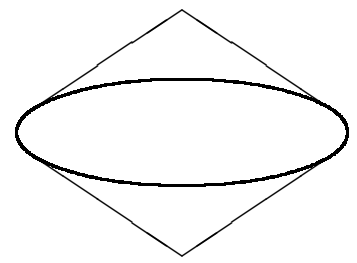

Figure 1: The set $S$

Let $C_{S}$ be the cone in $\mathbb{R}^{4}$ generated by $S$, so,

$$
C_{S}=\{\lambda \varphi: \varphi \in S \text { and } \lambda \geq 0\}
$$

Then $C_{S}$ is a closed cone with non-empty interior. Let $K_{S}$ be the dual of $C_{S}$, so $K_{S}^{*}=C_{S}$. Note that $u=(0,0,0,1) \in \mathbb{R}^{4}$ is in the interior of $K_{S}$, as $\varphi(u)>0$ for all $\varphi \in C_{S} \backslash\{0\}$. Now consider $\Sigma=\left\{\varphi \in C_{S}: \varphi(u)=1\right\}$ and remark that $\Sigma=S$. It follows that

$$
\Lambda=\{(0,0,-1,1),(0,0,1,1)\} \cup\{(\cos \vartheta, \sin \vartheta, 0,1): 0 \leq \vartheta<2 \pi\},
$$

which is a closed set.

Take $x=(0,0,1,0)$ and $y=(0,1,0,0)$. With the aid of Theorem 10 and [6, Theorem 4.1] it is easy to verify that $x \perp y$ and that both $x$ and $y$ are not in $K$. We now show that there are no nontrivial disjoint elements in $K_{S}$. Suppose that $x, y \in K_{S}, x \neq 0$, and $x \perp y$. Let $s_{x}=\{\varphi \in \bar{\Lambda}: \varphi(x)=0\}$ and $s_{y}=\{\varphi \in \bar{\Lambda}: \varphi(y)=0\}$. Then $s_{x} \cup s_{y}=\bar{\Lambda}$. As each $z \in K_{S} \backslash\{0\}$ with $\varphi(z)=0$ for some $\varphi$ on the relative boundary $\partial \Sigma$ of $\Sigma$ in the hyperplane $\{\varphi: \varphi(u)=1\}$ corresponds to a supporting hyperplane of $\Sigma$, we see from Figure 1 that there are, up to multiplication of the third coordinate by -1 , three cases to consider: (1) $s_{x}=\{(0,0,1,1)\},(2) s_{x}=\{(0,0,1,1),(\cos \vartheta, \sin \vartheta, 0,1)\}$ for some $\vartheta \in[0,2 \pi)$, and $(3) s_{x}=\{(\cos \vartheta, \sin \vartheta, 0,1)\}$ for some $\vartheta \in[0,2 \pi)$. In each of these cases $s_{y} \supseteq \operatorname{span}\left(\left\{\varphi: \varphi \in \bar{\Lambda} \backslash s_{x}\right\}\right)=\mathbb{R}^{4}$, which implies that $y=0$.

We can also use the functional representation to derive a geometric condition on $\partial \Sigma$ that ensures that a finite dimensional partially ordered vector space is an 
anti-lattice. In the statement of the result $V^{*}=\left\{\varphi \in X^{*}: \varphi(u)=1\right\}, \partial \Sigma$ is the relative boundary of $\Sigma$ in $V^{*}$, and $B_{\varepsilon}(\nu)=\left\{\varphi \in V^{*}:\|\varphi-\nu\|<\varepsilon\right\}$. We also use the fact that the dual cone $K^{*}$ of a closed generating cone $K$, i.e., $X=K-K$, is also generating in $X^{*}$, see e.g. [17, IV.1.1 and Krein's theorem IV.5.1].

Theorem 15. If $(X, K)$ is a finite dimensional Archimedean partially ordered vector space with an order unit $u$, and there exist $\nu \in \bar{\Lambda}$ and $\varepsilon>0$ such that

$$
B_{\varepsilon}(\nu) \cap \partial \Sigma \subseteq \bar{\Lambda},
$$

then $(X, K)$ has no non-trivial disjoint elements, and hence it is an anti-lattice.

Proof. For $S \subseteq V^{*}$ let aff $(S)$ denote the affine span of $S$ in $V^{*}$, so aff $(S)$ is the smallest affine subspace of $V^{*}$ containing $S$. We first show that

$$
\operatorname{aff}\left(B_{\varepsilon}(\nu) \cap \partial \Sigma\right)=V^{*} .
$$

Let $h: V^{*} \rightarrow \mathbb{R}$ be a supporting hyperplane at $\nu$, so $h(\nu)=0$ and $h(\varphi) \geq 0$ for all $\varphi \in \Sigma$. Then $\Sigma \cap h^{-1}(\{0\}) \subseteq \partial \Sigma$. We claim that there exists $\alpha>0$ such that

$$
\{\varphi \in \Sigma: h(\varphi) \leq \alpha\} \subseteq B_{\varepsilon}(\nu) .
$$

For the sake of contradiction suppose that there exist a sequence $\alpha_{k} \downarrow 0$ and $\rho_{k} \in X^{*}$ with $\left\|\rho_{k}\right\|=1$ such that $\nu-\varepsilon \rho_{k} \in \Sigma \cap \partial B_{\varepsilon}(\nu)$ and $h\left(\nu-\varepsilon \rho_{k}\right)=\alpha_{k}$. By taking a subsequence we may assume that $\rho_{k} \rightarrow \rho$ with $\|\rho\|=1$. Note that $\nu-\varepsilon \rho \in \Sigma$. Using the continuity of $h$ we see that $h(\nu-\varepsilon \rho)=0$ and hence $t \nu+(1-t)(\nu-\varepsilon \rho) \in \partial \Sigma$ for all $0 \leq t \leq 1$, which contradicts the assumption.

Let $\Delta=\{\varphi \in \Sigma: h(\varphi) \leq \alpha\}$ and denote its extreme points by $\operatorname{ex}(\Delta)$. By the claim $\operatorname{ex}(\Delta)$ is contained in $B_{\varepsilon}(\nu) \cap \partial \Sigma$, and hence in $\bar{\Lambda}$. Note that for each $\varphi \in \Sigma$ there exists $0<\lambda_{\varphi}<1$ such that $\left(1-\lambda_{\sigma}\right) h(\nu)+\lambda_{\varphi} h(\varphi) \leq \alpha$, and hence

$$
\operatorname{aff}(\Delta)=\operatorname{aff}(\Sigma)=V^{*} .
$$

So, $\Delta$ is a closed convex set with non-empty interior in $V^{*}$. By Carathéodory's theorem $\Delta$ is the convex hull of $\operatorname{ex}(\Delta)$. It follows that

$$
V^{*} \supseteq \operatorname{aff}\left(B_{\varepsilon}(\nu) \cap \partial \Sigma\right) \supseteq \operatorname{aff}(\Delta)=V^{*},
$$

which proves (4).

To prove the theorem we let $x \perp y$ in $(X, K)$ and $x \neq 0$, and need to show that $y=0$. Due to Theorem 10 and [6, Theorem 4.1] we have $\varphi(x) \varphi(y)=0$ for all $\varphi \in \bar{\Lambda}$. Let $s_{x}=\{\varphi \in \bar{\Lambda}: \varphi(x)=0\}$. If $s_{x}$ is empty, then $\varphi(y)=0$ for all $\varphi \in \bar{\Lambda}$, which implies that $y=0$, as $K^{*}$ is generating. Note also that $\operatorname{aff}\left(s_{x}\right) \neq V^{*}$, as otherwise $\varphi(x)=0$ for all $\varphi \in \bar{\Lambda}$, which is impossible as $x \neq 0$ and $K^{*}$ is generating.

We first consider the case where $\nu \notin \operatorname{aff}\left(s_{x}\right)$. Without loss of generality we may assume that $\varepsilon>0$ is so small that $B_{\varepsilon}(\nu) \cap$ aff $\left(s_{x}\right)$ is empty. As $\Delta \subseteq B_{\varepsilon}(\nu)$, it follows that $\Delta \cap \operatorname{aff}\left(s_{x}\right)$ is empty. So each $\varphi \in \operatorname{ex}(\Delta) \subseteq \bar{\Lambda}$ satisfies $\varphi(y)=0$. Thus, if we let $s_{y}=\{\varphi \in \bar{\Lambda}: \varphi(y)=0\}$, then

$$
V^{*}=\operatorname{aff}(\operatorname{ex}(\Delta)) \subseteq \operatorname{aff}\left(s_{y}\right) \subseteq V^{*} .
$$

This implies that $y=0$, as $K^{*}$ is generating. 
Now suppose that $\nu \in \operatorname{aff}\left(s_{x}\right)$. As aff $\left(s_{x}\right) \neq V^{*}$ and $\operatorname{aff}(\operatorname{ex}(\Delta))=V^{*}$, there exists $\psi \in \operatorname{ex}(\Delta)$ such that $\psi \notin \operatorname{aff}\left(s_{x}\right)$. Let $\delta>0$ be so small that $B_{\delta}(\psi) \subseteq B_{\varepsilon}(\nu)$ and $B_{\delta}(\psi) \cap$ aff $\left(s_{x}\right)$ is empty. Then $B_{\delta}(\psi) \cap \partial \Sigma \subseteq \bar{\Lambda}$. Replacing $\nu$ with $\psi$ and $\varepsilon$ with $\delta$ in the previous argument now shows that $y=0$.

Note that if $(X, K)$ is a finite dimensional Archimedean partially ordered vector space with an order unit $u$ whose dual cone $K^{*}$ is strictly convex, then $\partial \Sigma=\bar{\Lambda}$, so that $(X, K)$ has no disjoint elements. Particular examples are the finite dimensional Lorentz cones $L_{n}$, as they are self-dual and strictly convex.

For the space $\left(V, \mathrm{Pos}_{n}\right)$ of symmetric matrices ordered by the cone of positive semi-definite matrices it can be shown for all $n \geq 3$ that $\partial \Sigma$ does not satisfy the condition in Theorem 15. (For $n=2$ it is well-known that $\left(V, \mathrm{Pos}_{2}\right)$ is orderisomorphic to $\left(\mathbb{R}^{3}, L_{3}\right)$, see e.g. [7].) Nevertheless we can use the functional representation to show for all $n$ that $\left(V, \mathrm{Pos}_{n}\right)$ has no non-trivial disjoint elements. As a consequence $\left(V, \operatorname{Pos}_{n}\right)$ is an anti-lattice, which recovers a well-know result by Kadison [12].

Proposition 16. There are no non-trivial disjoint elements in $\left(V, \mathrm{Pos}_{n}\right)$.

Proof. See Section 5 for the computation of $\Sigma, \bar{\Lambda}$, and $\Phi$. Let $A, B \in V \backslash\{0\}$. By Theorem 10 and [6, Theorem 4.1] $A \perp B$ is equivalent to $\Phi(A) \perp \Phi(B)$. Remark that

$$
\begin{aligned}
\Phi(A) \perp \Phi(B) & \Longleftrightarrow|\operatorname{tr}(A C)| \wedge|\operatorname{tr}(B C)|=0 \text { for all } C \in \Lambda \\
& \Longleftrightarrow\left|\operatorname{tr}\left((A q) q^{\top}\right)\right| \wedge\left|\operatorname{tr}\left((B q) q^{\top}\right)\right|=0 \text { for all } q \in S^{n-1} \\
& \Longleftrightarrow|\langle A q, q\rangle| \wedge|\langle B q, q\rangle|=0 \quad \text { for all } q \in S^{n-1} .
\end{aligned}
$$

As $A \neq 0$ and $A$ is symmetric, it follows from the spectral theorem that $A$ has an eigenvalue $\lambda \neq 0$ with a normalised eigenvector $s$. Likewise $B$ has an eigenvalue $\mu \neq 0$ with normalised eigenvector $t$. Write $q=\alpha s+\beta t$ with $\alpha, \beta \in \mathbb{R}$. Then

$$
\langle A q, q\rangle=\langle\lambda \alpha s+\beta A t, \alpha s+\beta t\rangle=\lambda \alpha^{2}+\alpha \beta\langle A t, s\rangle+\lambda \alpha \beta\langle s, t\rangle+\beta^{2}\langle A t, t\rangle
$$

and

$$
\langle B q, q\rangle=\mu \beta^{2}+\alpha \beta\langle B s, t\rangle+\mu \alpha \beta\langle s, t\rangle+\alpha^{2}\langle B s, s\rangle .
$$

If $\langle B s, s\rangle \neq 0$, then $|\langle A s, s\rangle| \wedge|\langle B s, s\rangle| \neq 0$. If $\langle B s, s\rangle=0$, we can take $\beta=1 / \alpha>0$ to get

$$
|\langle A q, q\rangle|=\left|\lambda \alpha^{2}+\langle A t, s\rangle+\lambda\langle s, t\rangle+\frac{1}{\alpha^{2}}\langle A t, t\rangle\right| \rightarrow \infty \quad \text { as } \alpha \rightarrow \infty,
$$

and

$$
\langle B q, q\rangle=\frac{\mu}{\alpha^{2}}+\langle B s, t\rangle+\mu\langle s, t\rangle \neq 0 \quad \text { for } \alpha \text { large. }
$$

Therefore, $|\langle A q, q\rangle| \wedge|\langle B q, q\rangle| \neq 0$ for some $q \in \mathbb{R}^{n}$. Thus $A$ and $B$ are not disjoint.

Acknowledgement. O. van Gaans acknowledges the support by a 'VIDI subsidie' (639.032.510) of the Netherlands Organisation for Scientific Research (NWO). 


\section{References}

[1] C. D. Aliprantis, O. Burkinshaw, Positive operators, Academic Press, Inc., Orlando, 1985

[2] E.M. Alfsen, Facial structure of compact convex sets, Proc. London Math. Soc. (3) 18(1968), 385-404.

[3] G. Buskes and A.C.M. van Rooij, The vector lattice cover of certain partially ordered groups, J.Austral. Math. Soc. (Series A) 54(1993), 352-367.

[4] N. Dunford and J.T. Schwartz, Linear Operators, Part 1: General Theory, Wiley Interscience, New York, 1957.

[5] J. Faraut and A. Korányi, Analysis on Symmetric Cones. Oxford Mathematical Monographs. The Clarendon Press, Oxford University Press, New York, 1994.

[6] O. van Gaans and A. Kalauch, Disjointness in partially ordered vector spaces, Positivity 10(2006) no. 3, 573-589.

[7] O. van Gaans and A. Kalauch, Ideals and bands in pre-Riesz spaces, Positivity 12(2008), no. 4, 591-611.

[8] O. van Gaans and A. Kalauch, Bands in pervasive pre-Riesz spaces, Oper. Matrices $\mathbf{2}(2008)$ no. 2, 177-191.

[9] M. van Haandel, Completions in Riesz space theory, Ph.D. thesis, University of Nijmegen, 1993.

[10] G. Jameson, Ordered linear spaces, LNM 141, Springer-Verlag, Berlin, 1970.

[11] R.V. Kadison, A representation theory for commutative topological algebra. Mem. Amer. Math. Soc., 1951, (1951). no. 7.

[12] R.V. Kadison, Order properties of bounded self-adjoint operators, Proc. Amer. Math. Soc. 2(3), (1951), 505-510.

[13] S. Kakutani, Concrete representation of abstract $(M)$-spaces (A characterization of the space of continuous functions), Ann. Math. 42 no. 4, 1941.

[14] M. Krein, Propriétés fondamentales des ensembles coniques normaux dans l'espace de Banach. C. R. (Doklady) Acad. Sci. URSS (N.S.) 28, (1940). 13-17.

[15] L. Nachbin, A characterization of the normed vector ordered spaces of continuous functions over a compact space. Amer. J. Math. 71, (1949). 701-705.

[16] A.L. Peressini, Ordered topological vector spaces. Harper \& Row, Publishers, New YorkLondon 1967.

[17] B.Z. Vulikh, Introduction to the Theory of Cones in Normed Spaces (Russian), Izdat. Kalinin Univ., Kalinin, 1977

\begin{abstract}
Anke Kalauch FR Mathematik Inst. f. Analysis TU Dresden 01062 Dresden Germany
\end{abstract}

Bas Lemmens

School of Mathematics, Statistics \& Actuarial Sciences University of Kent CT27NF, Canterbury

United Kingdom

B. Lemmens@kent.ac.uk
Onno van Gaans

Mathematical Institute

Leiden University

P.O. Box 9512

2300 RA Leiden

The Netherlands

vangaans@math.leidenuniv.nl 